J. Lake Sci.(湖泊科学), 2009, 21(5): 663-668

http://www.jlakes.org. E-mail: jlakes@niglas.ac.cn

(C2009 by Journal of Lake Sciences

\title{
模拟水体 $\mathrm{pH}$ 控制条件下太湖梅梁湾沉积物中磷的释放特征 ${ }^{*}$
}

\author{
袁和忠 ${ }^{1,2}$, 沈 吉 $^{1}$, 刘恩峰 ${ }^{1}$, 孟祥华 ${ }^{3}$, 王建军 ${ }^{1}$ \\ (1: 中国科学院南京地理与湖泊研究所湖泊与环境国家重点实验室, 南京 210008) \\ (2: 中国科学院研究生院, 北京 100049) \\ (3: 济南大学城市发展学院, 济南 250022)
}

摘 要: 采用缓冲溶液控制水体 $\mathrm{pH}$ 值, 模拟研究了太湖梅梁湾沉积物扰动悬浮状态下水体磷的释放规律 (48h), 分析了实验 前后沉积物中磷的赋存形态组成及变化特征. 结果表明, $\mathrm{pH}$ 条件对沉积物磷释放具有显著影响, 强酸性 $(\mathrm{pH}=2.0)$ 及碱性 $(\mathrm{pH}=9.5,11.8)$ 条件下皆利于磷的释放，但强酸性水体磷含量随时间逐渐降低，而碱性水体磷呈持续释放状态; 中性条件 $(\mathrm{pH}=7.2)$ 磷的释放释放作用不明显. 磷释放达到稳定状态 $(14 \mathrm{~h})$ 后，碱性条件下 $(\mathrm{pH}=9.5,11.8)$ 磷释放量为酸性 $(\mathrm{pH}=2.0,4.6)$ 及中 性 $(\mathrm{pH}=7.2)$ 条件下的 4 倍以上. 通过对比释放前、后沉积物中磷的形态组成可知, 酸性条件主要为钻结合态磷的释放; 碱性条 件主要是铁结合态磷释放, 部分可发生再沉淀, 转化为钲结合态磷. 研究表明, 梅梁湾沉积物中铁结合态磷的解析是沉积物 磷的主要释放源, 是水体内源磷增加的重要贡献者.

关键词: 沉积物; 磷释放; 磷形态; $\mathrm{pH}$; 太湖

\section{Characteristic of phosphorus release with the control of $\mathrm{pH}$ of sediments from Meiliang Bay, Lake Taihu}

YUAN Hezhong ${ }^{1,2}$, SHEN Ji ${ }^{1}$, LIU Enfeng ${ }^{1}$, MENG Xianghua ${ }^{3} \&$ WANG Jianjun ${ }^{1}$

(1: State Key Laboratory of Lake Science and Environment, Nanjing Institute of Geography and Limnology, Chinese Academy of Sciences, Nanjing 210008, P.R.China)

(2: Graduate School of the Chinese Academy of Sciences, Beijing 100049, P.R.China)

(3: School of City Development, University of Jinan, Jinan 250022, P.R.China)

Abstract: The phosphorus stored in the sediments can be released into the overlying water, acting as internal source for lakes. pH is one of the important factors influencing phosphorus release. In this paper, phosphorus fractions and the effect of $\mathrm{pH}$ on $\mathrm{P}$ release (48h) were investigated for the sediment from Meiliang Bay, Lake Taihu. The $\mathrm{pH}$ of the system was controlled with different buffer solutions. The release characteristics of phosphorus were influenced markedly by the $\mathrm{pH}$ condition. Specifically, acidity ( $\mathrm{pH}=2.0)$ and alkalinity $(\mathrm{pH}=9.5,11.8)$ conditions were more beneficial for phosphorus release than the neutral $\mathrm{pH}(\mathrm{pH}=7.2)$ condition. The amount of phosphorus released from the sediments under alkalinity conditions $(\mathrm{pH}=9.5,11.8)$ was about 4 folds than that in the acidity $(\mathrm{pH}=2.0,4.6)$ and neutral condition when the release of phosphorus tended to be stable $(14 \mathrm{~h})$. There had been distinct difference for the phosphorus speciation in the sediments of different simulation system. With the analysis to the phosphorus speciation of sediments after release experiment, it can be concluded that acidic condition can enhance Ca-bound phosphorus release, whereas alkaline condition can promote the release of Fe-bound phosphorus significantly. Fe-bound phosphorus is the key form of phosphorus release in Meiliang Bay of Lake Taihu. $\mathrm{pH}$ value is an important mechanism of influencing phosphorus content and eutrophication. Keywords: Sediments; phosphorus release; phosphorus speciation; $\mathrm{pH}$; Lake Taihu

* 中国科学院知识创新工程重大项目(KZCX1-YW-14-5)、国家重点基础研究发展计划(973)(2008CB418104)和江苏省自 然科学基金项目(BK2008055)联合资助. 2008-10-27 收稿; 2009-02-12 收修改稿. 袁和忠, 男, 1981 年生, 博士研究生; E-mail: yuanhezhong@126.com 
富营养化是我国众多湖泊面临的主要环境问题之一, 磷作为藻类生长的主要的限制性营养盐 ${ }^{[1-2]}$, 浓 度过高被认为是水体富营养化的主要原因 ${ }^{[3-4]}$. 湖泊沉积物是磷等营养物质的重要蓄积库 ${ }^{[4]}$, 同时又是潜 在的二次污染源 ${ }^{[5-6]}$. 在长江中下游湖泊水体磷的环境背景值较高的情况下, 磷的内源负荷受到越来越多 的关注 ${ }^{[7-9]}$.

沉积物内源磷的释放受控于众多环境因素 ${ }^{[10-11]}$, 在不同 $\mathrm{pH}$ 值、氧化还原条件、微生物、沉积物扰动 状况等物理、化学及生物作用下 ${ }^{[12]}$, 表现出不同的迁移转化特征.其中上覆水的 $\mathrm{pH}$ 条件是影响磷迁移转 化的重要参数之一 ${ }^{[1,13]}$. 研究表明, 湖泊水华爆发过程中, 水土界面的 $\mathrm{pH}$ 条件发生显著的改变, 从而影 响磷在水土界面的生物地球化学循环过程 ${ }^{[14]}$. Jensen 等 ${ }^{[15]}$ 调查了丹麦不同湖泊 $\mathrm{pH}$ 值与 $\mathrm{PO}_{4}{ }^{3}$ 含量之间的 关系, 发现部分湖泊高 $\mathrm{pH}$ 值明显促进溶解活性磷(SRP)的释放; Jin 等 ${ }^{[5]}$ 通过添加酸碱控制实验水体 $\mathrm{pH}$ 值, 室内模拟研究了太湖不同湖区沉积物的磷释放量, 提出水体磷的含量与 $\mathrm{pH}$ 值存在明显的呼应关系. 但未 见有对释放过程进行详细的分析，同时后者单纯以酸碱调节水体 $\mathrm{pH}$ 值，可能引发室内实验水体在连续取 样过程中 $\mathrm{pH}$ 值产生较大的变幅. 太湖作为大型浅水湖泊, 沉积物易于受风浪扰动作用发生再悬浮, 促进 了内源磷营养盐向上覆水体的释放 ${ }^{[16-17]}$, 从而增加了水华爆发的风险. 本文选取磷内源负荷较重的太湖 梅梁湾表层沉积物, 通过添加缓冲溶液的方法严格控制水体的 $\mathrm{pH}$ 条件, 室内模拟研究一定程度扰动状态 下不同 $\mathrm{pH}$ 条件水体沉积物中磷的释放过程, 试图对水体沉积物磷释放的 $\mathrm{pH}$ 效应进行进一步的探讨.

\section{1 材料和方法}

\section{1 样品采集与分析}

梅梁湾位于太湖北部, 平均水深约 $2 \mathrm{~m}, 50 \%$ 为软泥覆盖, 松软沉积物厚度 $20 \mathrm{~cm}$ 以上 ${ }^{[15-16]}$, 风浪作用 较强烈, 沉积物再悬浮频繁 ${ }^{[16-17]}$. 水体营养盐含量较高, 整个湖区呈不同程度富营养化 ${ }^{[16]}$. 本次研究选 择梅梁湾北部靠近梁溪河口的湖区 $\left(31^{\circ} 27^{\prime} 05^{\prime \prime} \mathrm{N}, 120^{\circ} 07^{\prime} 49^{\prime \prime} \mathrm{E}\right.$ ) (水体 $\mathrm{pH}$ 为 7.2 ), 采用自制重力采样器无扰 动采集沉积物柱 3 份, 取表层 $0-2 \mathrm{~cm}$ 样品置于聚乙烯密封袋中充分混合, 迅速带回实验室后于 $4^{\circ} \mathrm{C}$ 冷 藏，部分低温烘干研磨过 150 目篮备用.

取适量湿样于坩埚中, 于 $105^{\circ} \mathrm{C}$ 下灼烧 $24 \mathrm{~h}$, 测得含水率. 沉积物有机物含量以 $550^{\circ} \mathrm{C}$ 高温灼烧 $2 \mathrm{~h}$ 所 得烧失量 (LOI) 计 ${ }^{[18-19]}$, 为 $3.4 \%$, 沉积物含水率为 $58.1 \%$. 沉积物粒度组成采用 Malvern 公司生产的 Mastersize-2000 激光粒度仪测得. $>64.0 \mu \mathrm{m}, 4.0-64.0 \mu \mathrm{m},<4.0 \mu \mathrm{m}$ 沉积物粒度组成分别为 $15.2 \%, 71.2 \%$, $13.6 \%$. 另取 $0.2 \mathrm{~g}$ 干样，首先以 $1 \mathrm{~mol} / \mathrm{L}$ 的 $\mathrm{MgCl}_{2}$ 溶液提取得弱吸附态磷，提取后的残渣参照 SMT 法分别 以 $\mathrm{NaOH}(1 \mathrm{~mol} / \mathrm{L}) 、 \mathrm{HCl}(1 \mathrm{~mol} / \mathrm{L})$ 、浓 $\mathrm{HCl}(3.5 \mathrm{~mol} / \mathrm{L})$ 为浸提剂分别对各形态磷进行提取 ${ }^{[3-4,20-21]}$. 提取液中 磷含量以钼锑抗分光光度法测定 ${ }^{[22-23]}$.

\section{2 磷释放实验}

磷的释放模拟过程中采用不同 $\mathrm{pH}$ 值缓冲溶液添加各种盐类配置人工湖水作为上覆水(表 1). 与单纯 以酸碱控制 $\mathrm{pH}$ 值相比，缓冲溶液能够保证模拟实验过程中 $\mathrm{pH}$ 值的相对稳定性. 为保持一定的离子浓度, 参考梅梁湾水体及其它湖泊主要离子的浓度特性, 人工湖水中加人的盐类的浓度为 $\mathrm{Na}_{2} \mathrm{SO}_{4} 0.15 \mathrm{~g} / \mathrm{L}$ 、 $\mathrm{NaHCO}_{3} 0.1 \mathrm{~g} / \mathrm{L} 、 \mathrm{NH}_{4} \mathrm{Cl} 0.05 \mathrm{~g} / \mathrm{L} 、 \mathrm{MgCl}_{2} 0.05 \mathrm{~g} / \mathrm{L}^{[7,17]}$. 分别取 $2.0 \mathrm{~g}$ 沉积物干样于雉形瓶中, 加配制好的人 工湖水定容至 $200 \mathrm{ml}$ 并置于磁力搅拌仪上 $\left(25 \pm 1{ }^{\circ} \mathrm{C}\right)$ 搅拌, 模拟过程中沉积物处于恰好完全悬浮状态. 分 别于 $1 、 2 、 4 、 6 、 10 、 14 、 18 、 24 、 36 、 48 \mathrm{~h}$ 采集混合水样测定溶解活性磷(SRP，以下水体磷含量皆以 $\mathrm{SRP}$ 计)浓度. 采样体积 $13 \mathrm{ml}$, 于 2500 转 $/ \mathrm{minn}$ 离心后取上清液 $10 \mathrm{ml}$, 剩余溶液与残渣转移到原雉形瓶中, 重新补充配置好的人工湖水至 $200 \mathrm{ml}$. 取出水样中磷含量测定采用钼锑抗分光光度法 ${ }^{[23]}$. 实验过程中 $\mathrm{pH}$ 的 变化在 0.0-0.5 之间, 变异系数为 0.00-0.16, 可以认为本次实验水体 $\mathrm{pH}$ 基本无变化, 满足实验条件(表 1).

\section{2 结果和讨论}

\section{1 不同 $\mathrm{pH}$ 值条件下上覆水体中磷的浓度变化特征}

由于模拟试验上覆水体中磷的初始浓度接近为 $0 \mathrm{mg} / \mathrm{L}$, 沉积物与上覆水营养盐浓度梯度大, 实验开 始的 $0-1 \mathrm{~h}$ 内, 沉积物中磷迅速释放. $1 \mathrm{~h}$ 时磷浓度按 $\mathrm{pH}$ 值从低到高分别为 $0.418 \mathrm{mg} / \mathrm{L} 、 0.127 \mathrm{mg} / \mathrm{L}$ 、 
$0.087 \mathrm{mg} / \mathrm{L} 、 0.249 \mathrm{mg} / \mathrm{L} 、 0.378 \mathrm{mg} / \mathrm{L}$ (图 1). 在初期的迅速释放后, 各 $\mathrm{pH}$ 值水体磷释放趋势呈现明显的分 化, 并表现出不同的浓度变化特征.

表 1 实验前后水体 $\mathrm{pH}$ 值变化

Tab.1 pH variation of overlying water before and after the experiment

\begin{tabular}{cccccc}
\hline 缓冲溶液 & $\mathrm{HCl}+\mathrm{KCl}$ & $\mathrm{NaAc}+\mathrm{HAc}$ & $\mathrm{Tris}+\mathrm{HCl}$ & $\begin{array}{c}\mathrm{Na}_{2} \mathrm{~B}_{4} \mathrm{O}_{7} \cdot 10 \mathrm{H}_{2} \mathrm{O} \\
+\mathrm{NaOH}\end{array}$ & $\begin{array}{c}\mathrm{NaOH}+\mathrm{NH}_{2} \mathrm{CH}_{2} \mathrm{COOH} \\
+\mathrm{NaCl}\end{array}$ \\
\hline 实验前 & 2.0 & 4.6 & 7.2 & 9.5 & 11.8 \\
实验后 & 2.5 & 4.5 & 7.2 & 9.5 & 11.3 \\
$\mathrm{pH}$ 变幅 $\left(C_{V}^{*}\right)$ & $0.5(0.16)$ & $0.1(0.02)$ & $0.0(0.00)$ & $0.0(0.00)$ & $0.5(0.03)$ \\
\hline
\end{tabular}

* $C_{V}$ 变异系数.

实验开始的 $1 \mathrm{~h}$ 时, 强酸性条件下上覆水中 磷含量明显高于其他各 $\mathrm{pH}$ 值条件磷含量. 随着 实验的进行, $\mathrm{pH}=2.0$ 的试样中磷浓度迅速降低直 至 $14 \mathrm{~h}$ 时趋于稳定, 磷浓度维持在 $0.111 \mathrm{mg} / \mathrm{L}$ 左 右. 弱酸性 $(\mathrm{pH}=4.6)$ 条件下水体磷浓度变化变化 与强酸性条件下磷的浓度变化趋势大致相似, 但 远较强酸性水体磷释放为缓, 由 $1 \mathrm{~h}$ 的 $0.127 \mathrm{mg} / \mathrm{L}$ 降低到 $10 \mathrm{~h}$ 时 $0.038 \mathrm{mg} / \mathrm{L}$, 并保持在 $0.038 \mathrm{mg} / \mathrm{L}$ 左 右. 碱性情况下, $1 \mathrm{~h}$ 内释放量低于强酸性, $1 \mathrm{~h}$ 后 磷含量呈逐步上升, $2 \mathrm{~h}$ 后皆高于强酸性水体磷含 量, 一直持续到实验结束, 且水体中磷的含量明 显高于酸性及中性 $\mathrm{pH}$ 值试样磷含量. 强碱性 $(\mathrm{pH}=11.8)$ 水体磷浓度由开始 $(1 \mathrm{~h})$ 时的 $0.378 \mathrm{mg} / \mathrm{L}$ 上升到实验结束时的 $0.564 \mathrm{mg} / \mathrm{L}$, 较弱碱性 $(\mathrm{pH}=9.48)$ 水体磷浓度由开始 $(1 \mathrm{~h})$ 时的 $0.249 \mathrm{mg} / \mathrm{L}$ 上升到实验结束时的 $0.394 \mathrm{mg} / \mathrm{L}$, 二者变化程度 类似, 但强碱性水体磷的含量一直保持高于较弱 碱性水体磷的含量. 中性 $\mathrm{pH}$ 值情况下整个实验 过程中磷的含量变化并不明显, 磷浓度一直维持 在约 $0.087 \mathrm{mg} / \mathrm{L}$.

实验开始阶段, 各 $\mathrm{pH}$ 值试样水体磷皆呈 急速释放, $1 \mathrm{~h}$ 内沉积物磷平均释放速率分别达

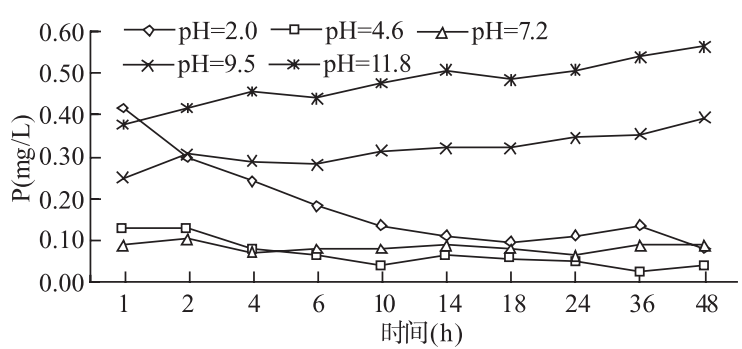

图 1 不同 $\mathrm{pH}$ 值上覆水中磷浓度变化

Fig.1 Phosphorus concentration variations of the overlying water with time at different $\mathrm{pH}$ value

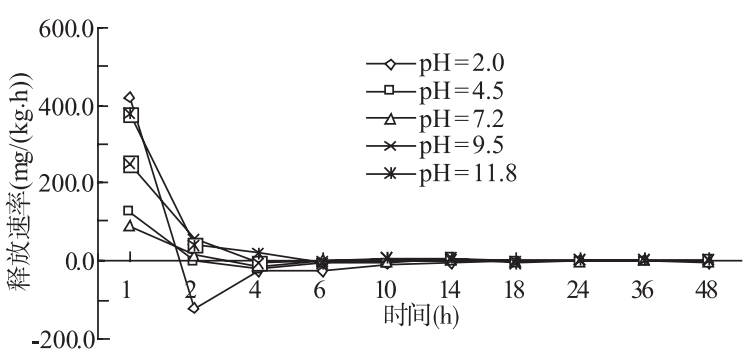

图 2 不同 $\mathrm{pH}$ 值沉积物中磷平均释放速率变化

Fig.2 Phosphorus mean release velocity variations with time at each $\mathrm{pH}$ value 到 $418.0 \mathrm{mg} /(\mathrm{kg} \cdot \mathrm{h}) 、 127.3 \mathrm{mg} /(\mathrm{kg} \cdot \mathrm{h}) 、 86.9 \mathrm{mg} /(\mathrm{kg} \cdot \mathrm{h}) 、 248.6 \mathrm{mg} /(\mathrm{kg} \cdot \mathrm{h}) 、 377.9 \mathrm{mg} /(\mathrm{kg} \cdot \mathrm{h}), 1 \mathrm{~h}$ 后皆呈不同趋势降 低(图 2). $4 \mathrm{~h}$ 后, 磷的平均释放速率均在 $21 \mathrm{mg} /(\mathrm{kg} \cdot \mathrm{h})$ 之下, 部分时段内表现为负值, 这是由于实验起始阶 段, 沉积物扰动状态下磷释放处于不稳定状态, 如强酸性水体中磷浓度 $1 \mathrm{~h}$ 内达到峰值, 接近中性 $\mathrm{pH}$ 水体 的 5 倍. 释放量达最大值后, 悬浮颗粒物的磷吸附开始逐渐占主导作用, 造成水体磷含量的降低, 释放速 率随之衰减, 当磷的吸附量大于释放量时, 表现为 $1 \mathrm{~h}$ 后沉积物磷释放速率为负值, $14 \mathrm{~h}$ 时磷的吸附-释放 达到动态平衡(图 2). 而碱性条件下, $1 \mathrm{~h}$ 内磷平均释放速率低于强酸性水体, 但都高于弱酸性及中性水体. $1 \mathrm{~h}$ 后随时间变化释放速率变缓, $6 \mathrm{~h}$ 后磷平均释放速率进一步趋缓, $14 \mathrm{~h}$ 时, $\mathrm{pH}=9.5 、 \mathrm{pH}=12$ 的试样磷释放 速率分别由初期(1h)的 $86.9 \mathrm{mg} /(\mathrm{kg} \cdot \mathrm{h}) 、 248.6 \mathrm{mg} /(\mathrm{kg} \cdot \mathrm{h})$ 降低至 $2.0 \mathrm{mg} /(\mathrm{kg} \cdot \mathrm{h})$ 左右并基本保持稳定状态，一直 持续到实验结束. 中性 $\mathrm{pH}$ 值条件下磷的释放无大的变化, 平均释放速率接近 $0 \mathrm{mg} /(\mathrm{kg} \cdot \mathrm{h})$.

除强酸性 $(\mathrm{pH}=2.0)$ 情况下初期形成磷的迅速释放，导致水体磷含量高于碱性情况，从实验开始 $2 \mathrm{~h}$ 一 
直到实验结束的 $48 \mathrm{~h}$, 碱性条件下水体磷的含量高于酸性与中性条件磷含量, 随时间呈现明显的一致性; 酸性 $\mathrm{pH}$ 条件下磷含量高于中性 $\mathrm{pH}$ 值磷含量. 中性 $\mathrm{pH}$ 值磷释放最为弱, 各 $\mathrm{pH}$ 值对磷含量的响应呈明显 的“U”型态(图 1), 这也与 $\mathrm{Jin}^{[5]}$ 、Nur ${ }^{[24]}$ 、王晓蓉 ${ }^{[25]}$ 等恒温震荡条件下的实验研究结果相吻合.

\section{2 沉积物中磷的形态变化}

沉积物磷形态研究方法较多 $[3,13,23,26]$, Ruban 将沉积物磷形态分为䥻结合态磷 (HCl-P)、铁结合态磷 (NaOH-P)、无机磷 (IP)、有机磷 (OP)及总磷(Conc.HCl-P)，这对探讨磷的来源具有良好的指示意义 ${ }^{[2,21]}$, 已 在诸多研究中得到广泛应用 ${ }^{[5,22,27]}$, 本次实验沉积物分析结果如表 2 所示. 无机磷的提取效率 $\left(\mathrm{MgCl}_{2}-\mathrm{P}+\right.$ $\mathrm{NaOH}-\mathrm{P}+\mathrm{HCl}-\mathrm{P}) / \mathrm{IP}$ )在 $97 \%-100.2 \%$ 之间，总磷的提取效率((IP+OP)/Conc.HCl-P)在 $70 \%-100.5 \%$ 之间, 除 $\mathrm{pH}=2.0$ 及 $\mathrm{pH}=11.8$ 提取效率略低外, 系统误差在较小的范围内(表 3). 分别对实验前后沉积物磷的形态进 行分析, 不同 $\mathrm{pH}$ 值各悬浮沉积物形态磷提取含量呈现显著的差异.

原泥样弱吸附态磷含量为 $18.34 \mathrm{mg} / \mathrm{kg}$, 占总磷比重最低, 仅约 $2.6 \%$. 各试样沉积物中弱吸附态的磷 $\mathrm{MgCl}_{2}-\mathrm{P}$ 含量为 $\mathrm{pH}=4.6>\mathrm{pH}=2.0>\mathrm{pH}=7.2>\mathrm{pH}=11.8>\mathrm{pH}=9.5$, 水体中可溶态磷部分源于弱吸附态的磷 的释放, 且酸性条件更利于弱吸附态磷的溶解释放.

NaOH-P 表征铁结合态磷(Fe-P), 各 pH 值水体沉积物中铁结合态磷皆低于原泥样铁结合态磷 (表 2), 说明各 $\mathrm{pH}$ 值水体中的铁结合态磷很大一部分已经以可溶态形式释放到水体中, 同时高 $\mathrm{pH}$ 值水体中铁结 合态磷值低于低 $\mathrm{pH}$ 值铁结合态磷值, 说明碱性及中性条件较酸性条件更利于水体中铁结合态磷的释放, 这主要是由于羟基与磷酸根竞争 $\mathrm{Fe}(\mathrm{III}) 、 \mathrm{Al}(\mathrm{III})$ 等氧化物及氢氧化物上的吸附点位而导致磷的释放 ${ }^{[28]}$.

HCl-P 表征钲结合态磷 (Ca-P), 钲结合态磷释放对 $\mathrm{pH}$ 不敏感 ${ }^{[29]}$, 表现在各 $\mathrm{pH}$ 值之间无显著差异, 但 皆远高于原泥样钙结合态磷值, 这可能归因于实验过程中其他形态磷向更稳定的、溶解度更小的䥻结合 态磷 (如磷灰石)的转化 ${ }^{[25]}$. 而碱性条件下䥻结合态磷主为高, 说明碱性条件更利于弱吸附态磷及铁、铅磷 向䥾磷的转化 ${ }^{[25]}$, 形成磷形态迁移转化的动态平衡.

表 2 释放前后悬浮沉积物磷的各种结合形态磷含量 $(\mathrm{mg} / \mathrm{kg})$

Tab.2 Phosphorus speciation concentration of suspended sediments in the process of experiment

\begin{tabular}{lllllll}
\hline 磷形态 & $\mathrm{pH}=2.0$ & $\mathrm{pH}=4.6$ & $\mathrm{pH}=7.2$ & $\mathrm{pH}=9.5$ & $\mathrm{pH}=11.8$ & 原泥样 \\
\hline $\mathrm{MgCl}_{2}-\mathrm{P}$ & 5.27 & 3.25 & 7.16 & 15.11 & 14.50 & 18.34 \\
$\mathrm{NaOH}-\mathrm{P}$ & 242.02 & 227.87 & 142.98 & 120.75 & 227.87 & 330.94 \\
$\mathrm{HCl}-\mathrm{P}$ & 237.97 & 213.72 & 233.93 & 254.14 & 250.10 & 72.24 \\
$\mathrm{IP}$ & 508.80 & 557.98 & 539.12 & 445.47 & 392.25 & 559.33 \\
$\mathrm{OP}$ & 166.23 & 152.08 & 157.13 & 132.88 & 131.87 & 159.15 \\
\hline Conc.HCl-P & 754.86 & 738.69 & 625.51 & 504.24 & 750.82 & 714.44 \\
\hline
\end{tabular}

表 3 释放前后悬浮沉积物磷的各种形态结合磷提取效率

Tab.3 Phosphorus speciation extraction efficiency of suspended sediments in the process of experiment

\begin{tabular}{lllllll}
\hline 磷形态 & $\mathrm{pH}=2.0$ & $\mathrm{pH}=4.6$ & $\mathrm{pH}=7.2$ & $\mathrm{pH}=9.5$ & $\mathrm{pH}=11.8$ & 原泥样 \\
\hline$\left(\mathrm{MgCl}_{2}-\mathrm{P}+\mathrm{NaOH}-\mathrm{P}+\mathrm{HCl}-\mathrm{P}\right) / \mathrm{IP}$ & 1.00 & 0.99 & 0.97 & 0.99 & 0.99 & 1.02 \\
$(\mathrm{IP}+\mathrm{OP}) / \mathrm{Conc} . \mathrm{HCl}-\mathrm{P}$ & 0.77 & 0.86 & 0.97 & 1.05 & 0.70 & 0.92 \\
\hline
\end{tabular}

无机磷包括铁结合态磷 ( Fe-P) 和钻结合态磷, 其含量为 $\mathrm{pH}=4.6>\mathrm{pH}=7.2>\mathrm{pH}=2.0>\mathrm{pH}=9.5>$ $\mathrm{pH}=11.8$, 说明碱性及强酸性皆利于无机磷的释放, 而碱性情况下无机磷释放量随碱性增强而增大. 有机 磷含量变化不明显, 实验前后含量在 $130.87-166.23 \mathrm{mg} / \mathrm{kg}$ 之间, 表明实验时间较短, 未促成有机磷的大规 模的转化. 其中碱性条件下 OP 值低于酸性及中性条件下的 OP 值, 这指示碱性条件相比酸性及中性情况 更利于有机磷向其它形态磷的转化, 其机理有待深人的探讨.

实验开始阶段 (1h), 强酸状态下水体中磷浓度最大, 主要为酸的溶解作用, 沉积物中部分䥻磷酸盐如 
差基磷灰石 $\left(\mathrm{Ca}_{10}\left(\mathrm{PO}_{4}\right)_{6}(\mathrm{OH})_{2}\right)$ 及弱吸附态磷等迅速释放 ${ }^{[29-30]}$, 即酸性条件有利于 HCl-P 及弱吸附态磷的 释放. 而碱性条件下, 由于配位体的交换作用, $\mathrm{OH}^{-}$与沉积物中磷酸根产生吸附点位的竞争 ${ }^{[5,28]}$, 使得沉 积物中铁、铝结合态磷大量解析，并呈持续释放状态. $\mathrm{NaOH}-\mathrm{P}$ 对 $\mathrm{pH}$ 值较敏感，高 $\mathrm{pH}$ 值条件下，与无定 形或弱晶形铁、铝盐如水铁矿 $(\mathrm{FeOOH}) 、$ 蓝铁矿 $\left(\mathrm{Fe}_{3}\left(\mathrm{PO}_{4}\right)_{2} \cdot\left(\mathrm{H}_{2} \mathrm{O}\right)_{8}\right)$ 、水铝石 $\left(\mathrm{Al}_{2} \mathrm{O}_{3} \cdot \mathrm{H}_{2} \mathrm{O}\right)$ 等结合吸附的磷开 始向上覆水体持续释放 ${ }^{[31]}$. 中性 $\mathrm{pH}$ 值条件下磷的释放无大的变化, 磷平均释放速率接近 $0 \mathrm{mg} /(\mathrm{kg} . \mathrm{h})$, 磷 的吸附与释放呈平衡状态, 这也与 Nur 和 Bates 的对美国俄克拉荷马州 Carl Blackwell 湖研究结果一致 ${ }^{[24]}$.

铁磷 $(\mathrm{Fe}-\mathrm{P}$ )在原泥样中所占比重最高, 相对比例达 $46.4 \%$ (表 2). 作为重要的生物可利用磷源, Fe-P 的 含量可用作指示环境污染程度的指标之一, 污染越重, 铁结合态磷的含量越高 ${ }^{[32-33]}$, 梅梁湾梁溪河口处 受纳大量的城市废水及农业灌溉漫流, 为重富营养区 ${ }^{[33]}$, 同时本区沉积物以细颗粒粉砂质土 $(4.0-64.0 \mu \mathrm{m})$ 为主, 含量约 $71.2 \%$, 小于 $64.0 \mu \mathrm{m}$ 粒径的颗粒含量为 $84.8 \%$, 利于铁结合态磷的吸附沉积 ${ }^{[34]}$, 使得铁结合 态磷含量高, 约占总磷含量的 $46.3 \%$. Fe-P/Ca-P 的值可作为鉴别沉积物酸化或碱化引起的磷释放的简单 指数 ${ }^{[20]}, \mathrm{Fe}-\mathrm{P} / \mathrm{Ca}-\mathrm{P}$ 的比值小于 0.5 的沉积物在低 $\mathrm{pH}$ 时可达到较高的磷释放速度. Fe-P/ Ca-P 的比值较高 时在较高 $\mathrm{pH}$ 值时可达到较高的磷释放速度. Jing 通过对太湖沉积物的研究中也提出类似的规律 ${ }^{[5]}$. 本次 实验所采原泥样 Fe-P/Ca-P 的比值约 4.6, 远大于 0.5 , 高 $\mathrm{pH}$ 值下有利于磷的释放, 本次磷释放实验碱性 条件过程中磷的高释放量证明了这一点.

\section{3 结论}

本次实验通过利用缓冲溶液 $\mathrm{pH}$ 值的相对稳定性配制人工湖水，探讨了连续状态下太湖北部梅梁湾 沉积物经较强扰动作用磷释放和不同 $\mathrm{pH}$ 值之间的关系, 结果表明:

(1)不同 $\mathrm{pH}$ 值水体沉积物释放磷的趋势差异明显. 碱性条件下，磷释放持续发生，上覆水的磷浓度 逐步升高, 除初期短时间(2h)内低于强酸性上体覆水磷含量外, 碱性水体磷浓度一直保持高于低 $\mathrm{pH}$ 值 水体的磷浓度, 强碱性高于弱碱性. $14 \mathrm{~h}$ 时碱性水体磷含量为酸性及中性 $\mathrm{pH}$ 值磷含量 4 倍以上, 且呈扩 大的趋势.

(2)碱性条件下沉积物中铁磷释放明显, 且呈持续释放态势, 导致上覆水中磷的浓度逐步升高. 碱性 越强, 磷释放量越大. 太湖水体总体呈弱碱性 ${ }^{[35]}$, 利于铁结合态磷的释放, 是湖泊内源磷增加的主要机 制之一, 为考量太湖磷释放潜力的重要指标.

致谢: 感谢中国科学院太湖湖泊生态系统研究站季江高级工程师在采样过程中提供的帮助.

\section{4 参考文献}

[1] Rydin E. Potentially mobile phosphorus in lake Erken sediment. Water Research, 2000, 34(7): 2037-2042.

[2] Herman G, Nishri A, Berman T. A long-term prediction model for total phosphorus concentrations in Lake Kinneret. Water Research, 1989, 23(1): 61-66.

[3] Ruban V, López-Sánchez JF, Pardo P et al. Harmonized protocol and certified reference material for the determination of extractable contents of phosphorus in freshwater sediments-A synthesis of recent works. Fresenius $J$ Anal Chem, 2001, 370: 224-228.

[4] Ruban V, Brigault S, Demare D et al. An investigation of the origin and mobility of phosphorus in freshwater sediments from Bort-Les-Orgues Reservoir, France. Journal of Environmental Monitoring, 1999, 1: 403-407.

[5] Jin XC, Wang SR, Pang Y et al. Phosphorus fractions and the effect of $\mathrm{pH}$ on the phosphorus release of the sediments from different trophic areas in Taihu Lake, China. Environmental Pollution, 2006, 139: 288-295.

[6] Lijklema L, Koelmans AA, Portielje R. Water quality impacts of sediment pollution and the role of early diagenesis. Water Science and Technology, 1993, 28: 1-12.

[7] 范成新, 羊向东, 史龙新等. 江苏湖泊富营养化特征、成因及解决途径. 长江流域资源与环境, 2005, 14(2): 218-223.

[8] 朱广伟. 太湖富营养化现状及原因分析. 湖泊科学, 2008, 20(1): 21-26.

[9] 汪家权, 孙亚敏, 钱家忠等. 巢湖底泥磷的释放模拟实验研究. 环境科学学报, 2002, 22(6): 738-742. 
[10] Gao L, Zhou JM, Yang H et al. Phosphosrus fractions in sediment profiles and their potential contributions to eutrophication in Dianchi lake. Environmental Geology, 2005, 48: 835-844.

[11] Pettersson K. Phosphorus characteristics of settling and suspended particles in Lake Erken. The Science of the Total Environment, 2001, 266: 79-86.

[12] Ullman WJ, Sandstrom MW. Dissolved nutrient fluxes from the nearshore sediments of Bowling Green Bay, central Great Barrier Reef Lagoon (Australia). Estuarine, Coastal and Shelf Science, 1987, 24: 289-303.

[13] Wang SR, Jin XC, Zhao HH et al. Phosphorus fractions and its release in the sediments from the shallow lakes in the middle and lower reaches of Yangtze River area in China. Colloids and Surfaces A:Physicochemical and Engineering Aspects, 2006, 273(1-3): 109-116

[14] Boström B, Andersen JM. Fleischer S et al. Exchange of phosphorus across the sediment-water interface. Hydrobiologia, 1988, 170: $229-244$

[15] Jensen HS, Andersen FØ. Importance of temperature, nitrate,and $\mathrm{pH}$ for phosphate release from aerobic sediments of four shallow Eutrophic lakes. Limnology \& Oceanography, 1992, 37(3): 577-589.

[16] 范成新, 张 路, 秦伯强等. 风浪作用下太湖悬浮态颗粒物中磷的动态释放估算. 中国科学(D 辑), 2003, 33(8): 760-768.

[17] 秦伯强, 胡维平, 高 光. 太湖沉积物悬浮的动力机制及内源释放的概念性模型. 科学通报, 2003, 48(17): 1822-1831.

[18] Selig U. Particle size-related phosphate binding and P release at the sediment-water interface in a shallow German lake. Hydrobiologia, 2003, 492: 107-118.

[19] Hirota J, Szyper JP. Separation of total particulate carbon into inorganic and organic components. Limnology \& Oceanography, 1975, 20(5): 896-900.

[20] 黄清辉, 王 否, 王子健. 中国湖泊水域中磷形态转化及其潜在生态效应研究动态. 湖泊科学, 2006, 18(3): 199-206.

[21] Ruban V, López-Sánchez JF, Pardo P et al. Selection and evaluation of sequential extraction procedures for the determination of phosphorus forms in lake sediment. Journal of Environmental Monitoring, 1999, 1: 51-56.

[22] Pardo P, López-Sánchez JF, Rauret G. Characterisation, validation and comparison of three methods for the extraction of phosphate from sediments. Analytica Chimica Acta, 1998, 376: 183-195.

[23] 金相灿, 屠清瑛. 湖泊富营养化调查规范(第二版). 北京: 中国环境科学出版社, 1990.

[24] Nur R, Bates MH. The effects of pH on the aluminum, iron and calcium phosphate fractions of lake sediments. Water Research, 1979, 13: 813-815.

[25] 王晓蓉, 华兆哲, 徐 菱等. 环境条件变化对太湖沉积物磷释放的影响. 环境化学, 1996, 15(1): 15-19.

[26] Hieltjes AHM, Lambertus L. Fractionation of inorganic phosphates in calcareous sediments. Journal of Environmental Quality, 1980, 9: 405-407.

[27] 黄清辉, 王东红, 王春霞等. 太湖梅梁湾和五里湖沉积物磷形态的垂向变化. 中国环境科学, 2004, 24(2): 147-150.

[28] Kim LH, Choi E, Stenstrom MK. Sediment characteristics, phosphorus types and phosphorus release rates between river and lake sediments. Chemosphere, 2003, 50: 53-61.

[29] 焦念志. 关于沉积物释磷问题的研究. 海洋湖沼通报, 1989, 2: 80-84.

[30] House WA, Denison FH. Factors influencing the measurement of equilibrium phosphate concentrations in river sediments. Water Research, 2000, 34(4): 1187-1200.

[31] Jensen HS, Thamdrup B. Iron-bound phosphorus in marine sediments as measured by bicarbonate-dithionite extraction. Hydrobiologia, 1993, 253: 47-59.

[32] Hisashi J. Fractionation of phosphorus and releasable traction in sediment mud of Osaka Bay. Bull Jap Soc Sci Fish, 1983, 49(3): 447-454.

[33] 张 路, 范成新, 池俏俏等. 太湖及其主要人湖河流沉积磷形态分布研究. 地球化学, 2004, 33(4): 423-432.

[34] 王圣瑞, 赵海超, 周小宁等. 五里湖与贡湖不同粒径沉积物中有机质、总氮和磷形态分布研究. 环境科学研究, 2004, 17(增刊): 11-14.

[35] 孙顺才, 黄渏平. 太湖. 北京: 海洋出版社, 1993. 